\title{
Mira Variables: Statistical Parallaxes, Kinematics and Period-Luminosity Relation
}

\author{
E. Kilpio, O. Malkov
}

Institute of Astronomy, 48 Pyatnitskaya Str., Moscow 109017, Russia

N. Kharchenko

Main Astrophysical Observatory, Golosiiv, 252650 Kiev, Ukraine

E. Schilbach

Astrophysikalisches Institut Potsdam, An der Sternwarte 16, D-14482

Potsdam, Germany

\begin{abstract}
Based on the statistical parallaxes, the mean absolute magnitudes and the spatial kinematical parameters were determined as functions of the periods for Mira variables given in the GCVS-4 and selected stars for which new kinematical data were obtained during the last decade. The $\mathrm{BC}$ were derived for the oxygen-rich Miras and the $P-L$ relation was compared with predictions from pulsation theory. The following findings result from this analysis:

1) With respect to Miras, the GCVS-4 is complete down to $V=9^{\mathrm{m}}$.

2) The kinematical and orbital parameters indicate a higher age for the oxygen-rich Miras pulsating with smaller periods.

3) The $M_{\text {bol }}$-period relation of the oxygen-rich Miras shows a low dispersion and does not depend on the pulsation mode. The resulting $P-L$ relation is a superposition of $P-L$ relations of different pulsation modes. 4) The fraction of the fundamental mode pulsators decreases with increasing pulsation period.
\end{abstract}

The 4-th edition of the General Catalog of Variable Stars, the GCVS-4 (Kholopov et al. 1985-1988) contains 5042 Miras with known periods. The radial velocities were taken from 9 different catalogues. The proper motions from the five catalogues were reduced to the Hipparcos reference system. The resulting list contains these data for about 1000 stars. For 392 Miras in our sample both the proper motions in the Hipparcos system and radial velocities are available.

The method of statistical parallax determination is based on the assumption that the peculiar motions of stars are randomly distributed and their proper motions, on the average, reflect the solar motion. According to their period, the oxygen-rich Miras were divided into six groups. The statistical parallaxes computed for each group by the five different methods did not differ significantly and were averaged.

Based on the statistical parallaxes, the absolute magnitudes were derived by standard procedure with extinction values taken from Sharov's (1963) map. 

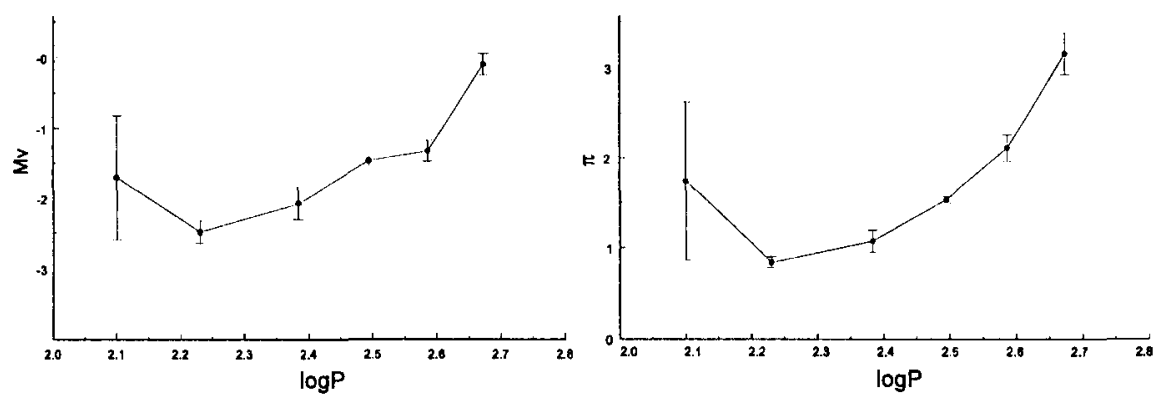

Figure 1. Absolute magnitude and mean statistical parallax versus period for the oxygen-rich Miras.

We computed the spatial velocity components and their dispersions in the Galactic rectangular system. The elements of Galactic box orbits, eccentricity and maximum $Z$-distance from the Galactic plane which a star can reach in its orbital motion, were obtained with an assumption of the Galactic potential form proposed by Saio \& Yoshii (1979).

The kinematical and orbital data indicate a higher age of the oxygen-rich Miras with smaller periods (an exception is the group with $P<139 \mathrm{~d}$ ). Smaller rotation velocities and larger velocity dispersions support the idea that these stars belong to older Galactic subsystems.

In order to compare the $P-L$ relation with theoretical predictions of the pulsation theory, we converted the averaged visual magnitudes to bolometric ones with bolometric correction $(B C): B C=-11.3( \pm 0.8) \log P+22.6( \pm 2.1)$. The derived relation shows a low dispersion and does not depend on the mode of pulsation.

The averaged mean bolometric magnitudes were computed for six groups of oxygen-rich Mira variables and the resulting $M_{\text {bol }}-\log P$ relation was compared with theoretical predictions of Barthès (1998) for near-solar metallicity. Our results show an increase of the luminosity with increasing $P$. The relation for $\log P>2.3$ is located in the area of theoretical $P-L$ relations for fundamental pulsators of different masses (Barthès 1998); but our relation is steeper than any of the theoretical ones.

Our results can be explained by a probable decrease of the fraction of fundamental pulsators with increasing period. Another possible explanation: a slight drift of the mean mass and/or metallicity with the $P$ in the considered sample may also cause a similar behavior of the $P-L$ relation.

\section{References}

Barthès, D. 1998, A\&A, 333, 647

Kholopov, P. N. et al. 1985-1988, General Catalogue of Variable Stars, 4th Edition, (Moscow: Nauka), [GCVS-4]

Saio, H. \& Yoshii, Y. 1979, PASP, 91, 553

Sharov, A. S. 1963, AZlı, 40, 900 